\title{
A Comparative Ultrastructural Study on Conidium Differentiation in the Cladosarum-like Mutant 22B of Aspergillus aureolatus
}

\author{
By R. VUJIČIĆ AND M. MUNTANJOLA-CVETKOVIĆ \\ Institute for Biological Research, 29 Novembar I42, I 1060 Belgrade 38, Yugoslavia
}

(Received 2 January 1973; revised 3I May 1973)

\begin{abstract}
SUMMAR Y
The ultrastructure of conidium formation in a wild-type, and in a temperaturesensitive and osmotic-remedial strain of Aspergillus aureolatus has been studied. In the mutant 22B the development of true conidia was blocked at non-permissive conditions. The conidial chain elongation was basipetal in the wild-type and apical in the mutant. The result of the apical elongation was a simple or a branched chain of undifferentiated conidia, encompassed by a thin single-layered non-pigmented wall, which were limited by septa with open pores. The last, or two last, elements in the chain were potential conidia, because if the temperature was lowered they could develop into true conidia. Their wall could complete the process of thickening and appeared three-layered. The outer wall layer acquired ornaments and pigment, and the septal pore was occluded.
\end{abstract}

\section{INTRODUCTION}

The importance of fine-structure studies of conidium ontogeny has been emphasized by several authors, and reports on conidiogenesis in various fungi have been published in an attempt to verify taxonomic groups or to gain better knowledge of the cellular events leading to conidium formation. In the Aspergilli two studies have been made of the fine structure of conidiogenesis (Tanaka \& Yanagita, I963; Trinci, Peat \& Banbury, 1968). Differences between mature and immature conidia of Aspergillus nidulans have been reported by Weisberg \& Turian (197I), but most ultrastructural studies have been made of dormant and germinating spores (Tanaka, 1966; Tsukahara, I968; Border \& Trinci, I970; Campbell, I97I; Florance, Denison \& Allen, 1972).

Conidiation in mutant 22B of Aspergillus aureolatus is temperature-sensitive and osmoticremedial (Muntanjola-Cvetković \& Nešković, I $972 a, b$ ). In the present study a comparison is made of conidium development at permissive and non-permissive temperatures.

\section{METHODS}

The organisms used were the wild-type 5BKz of Aspergillus aureolatus and its mutant 22B-3W (Muntanjola-Cvetković \& Nešković, 1972a).

The nutrient media employed were Czapek's solution agar plus different sugar concentrations, and Potato-Dextrose-Agar, $\mathrm{pH} \mathrm{6.5}$. The colonies were grown at $17^{\circ}, 22^{\circ}$, and $24^{\circ} \mathrm{C}$ for 10 and 20 days.

For electron microscopy, the material removed from these colonies was fixed in $2 \%(\mathrm{w} / \mathrm{v})$ potassium permanganate in a phosphate buffer $(\mathrm{pH} \mathrm{7.4)}$ ) at room temperature for $2 \mathrm{~h}$. After 
fixation the material was dehydrated in ethanol series and embedded in Araldite. Sections were cut with a diamond knife, stained, and examined under a Siemens Elmiscop IOI electron microscope.

\section{RESULTS}

The growth of the vesicle was the same in the wild-type and in the mutant, and followed the pattern described by Raper \& Fennell (1965).

In the wild-type, a primary series of sterigmata or metulae were produced by the vesicle, which subsequently formed a secondary series of sterigmata or phialides (Fig. I). The thinwalled sterigmata contrasted with the thick-walled vesicle; only the tapered end of the phialides was thickened (Fig. 2). The conidium initial originated as a bud at the apex of the phialide (Fig. 3). The phialide wall ruptured at the apex allowing the conidial initial to emerge. The broken wall formed a rim in which three layers of different electron-densities were observed: the outer and the inner layer were electron-dense and contrasted with the electron-transparent middle layer (Fig. 4). The outer and the middle layer were not involved in conidiation, and only the inner one was active during conidium formation (Fig. 4). The conidium initial expanded, and when it had attained a certain size a septum was formed by invagination of the plasma membrane. Wall synthesis was apparently intense on the conidium side, and a differential thickening occurred and resulted ultimately in the separation of the spore from the phialide (Fig. 4). The distal tapered end of the phialide continued its activity, and successive spores emerged. Ultimately, the conidium wall consisted of three layers (Fig. 5) which Florance et al. (1972) identified as $\mathrm{D}_{1}, \mathrm{D}_{2}$, and $\mathrm{D}_{3}$ for dormant conidia of Aspergillus nidulans.

In the mutant 22B, chain elongation of the pseudo-conidia, formed at non-permissive temperatures, differed from the above and was more comparable to the mechanism of hyphal growth. Each new pseudo-conidium was formed by the swelling of the distal end of the previous one. Before the 'conidial' initial attained its ultimate size, it also started to bud at its apex (Fig. 6). This finally resulted in the formation of a chain of elements of varied shape and size, encompassed by a continuous, single-layered wall lacking pigment. The cytoplasm of each pseudo-conidium contained a single nucleus, a well-developed endoplasmic reticulum, mitochondria, a few lipid inclusions and abundant small vacuoles filled with electrontransparent contents (Figs. 7 to II). Endoplasmic reticulum with a series of tubular fragments and vesicles were observed to be much more developed in the pseudo-conidia than in true mature condia. The nuclear division and the migration of the apical daughternucleus to the new-forming cell occurred before the septum formed (Figs. 6 and II). The zone of the septum was bordered by the endoplasmic reticulum, and the Woronin bodies were always found in the vicinity of the septal pore. Through the septal pores there was a continuity of the protoplasm from the vesicle to the last 'conidium' in the chain.

In the colonies of the mutant 22B which were first grown at $26^{\circ} \mathrm{C}$ and then transferred to $18{ }^{\circ} \mathrm{C}$, the young pseudo-conidial elements underwent a series of changes: the apical growth ceased, and the last elements of the chain acquired the characteristics of true conidia (Figs. I2 and I3); the conidial wall became thicker, forming two or three layers, and the outer layer of the wall acquired ornamentations and pigmentation typical of the true conidia; a differential thickening was observed on the inner side of the conidium, and the septal pore became occluded; finally, detachment followed this stage. 

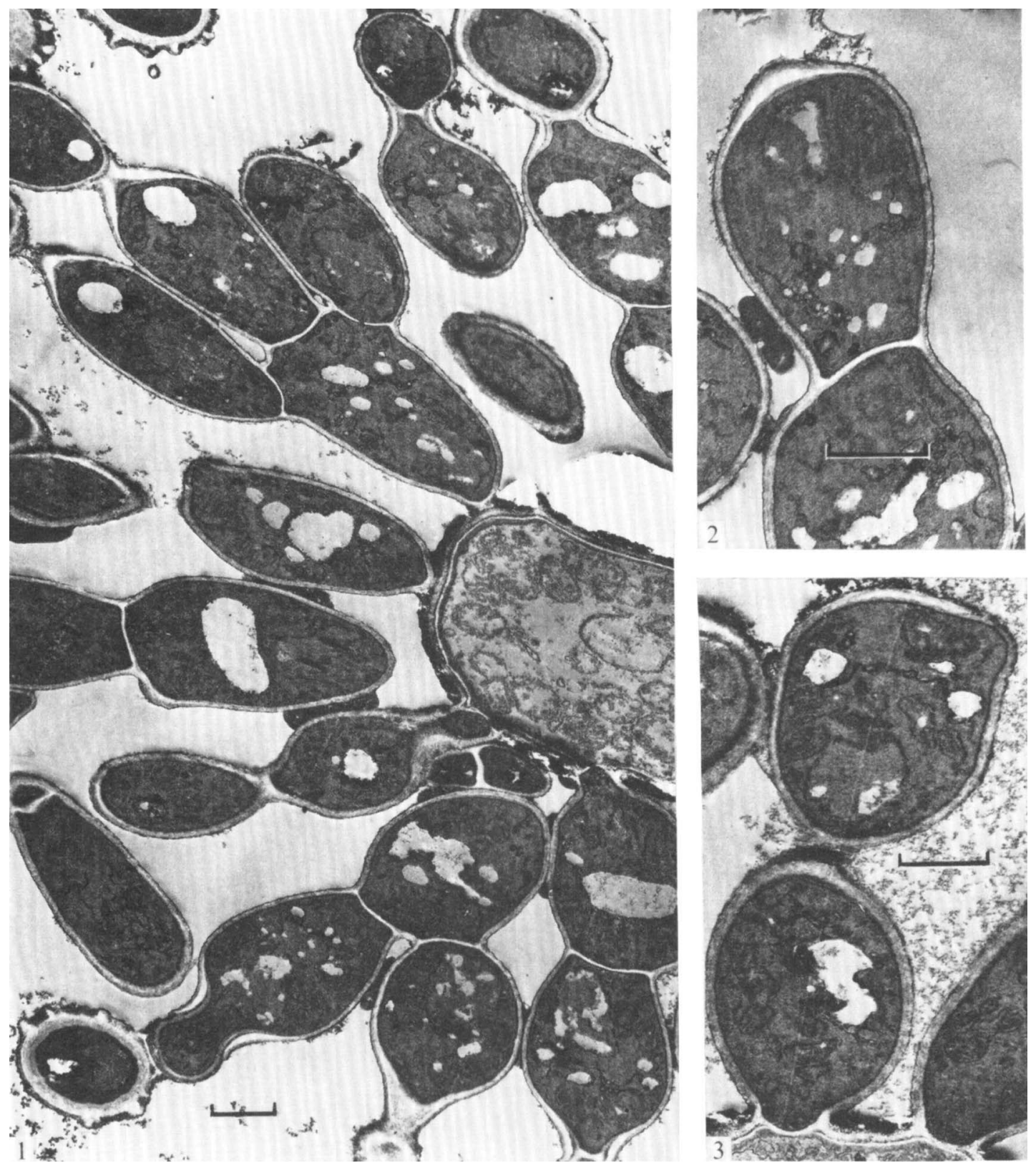

Fig. I. Aspergillus aureolatus, wild-type. Thin section of a vesicle, with metulae and phialides; the phialides are arranged in verticils of 2 to 3 elements.

Fig. 2. The tapered end of the phialide showing its thickened wall.

Fig. 3. Budding at the apex of a phialide.

(Bar markers represent $\mathrm{I} \mu \mathrm{m}$.) 

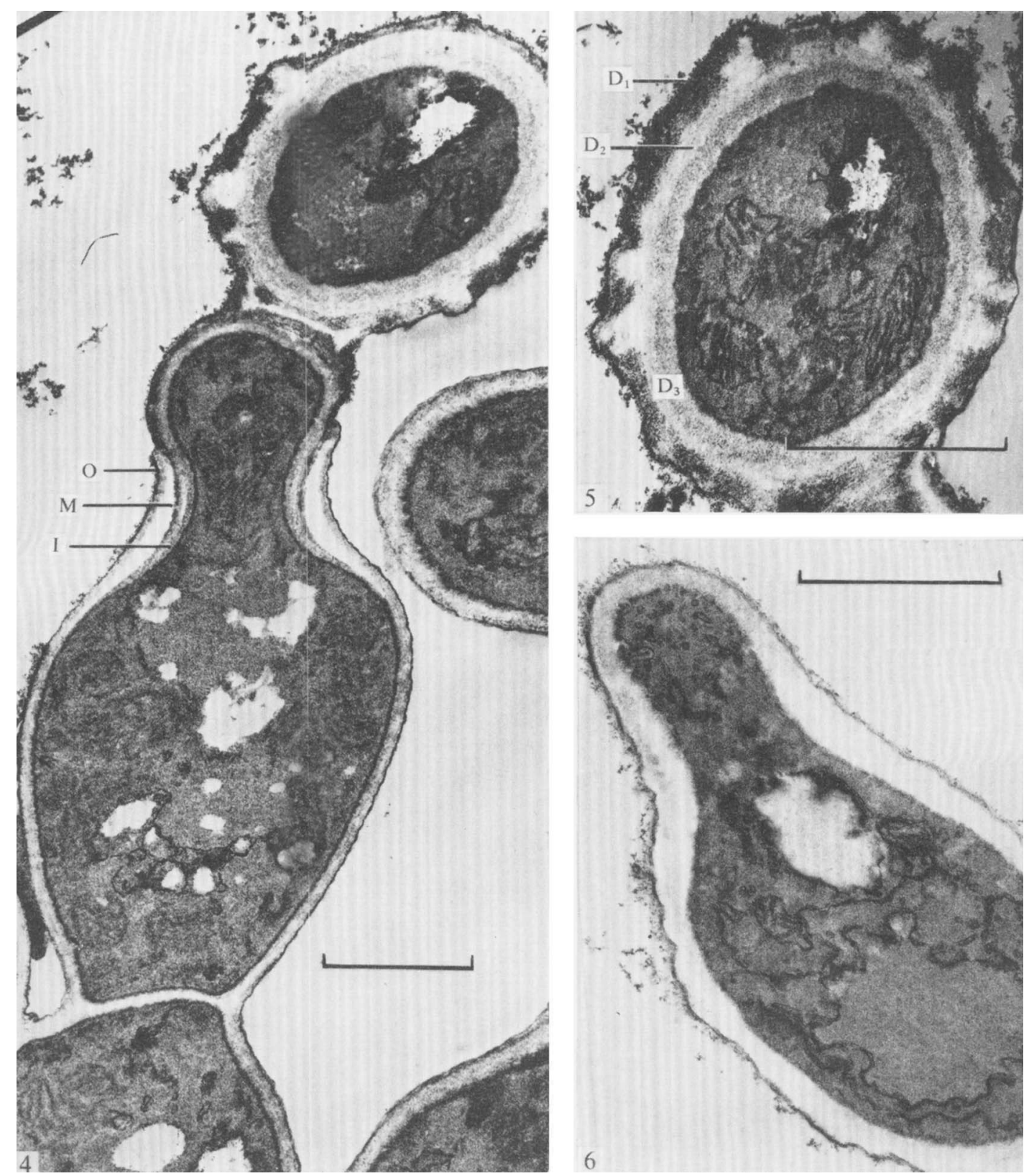

Fig. 4. Aspergillus aureolatus, wild-type. Longitudinal section through a phialide with an alreadyformed conidium and the primordium of a new one. Note the three layers of the rim: $\mathrm{O}$, outer layer; M, middle layer; I, inner layer.

Fig. 5. Thin section of a mature conidium with its three layered wall: $D_{1}, D_{2}$ and $D_{3}$.

Fig. 6. Mutant 22B. Apical extension of the last element of a pseudo-conidial chain, with abundant vesicles near the apex.

(Bar markers represent $\mathrm{I} \mu \mathrm{m}$.) 

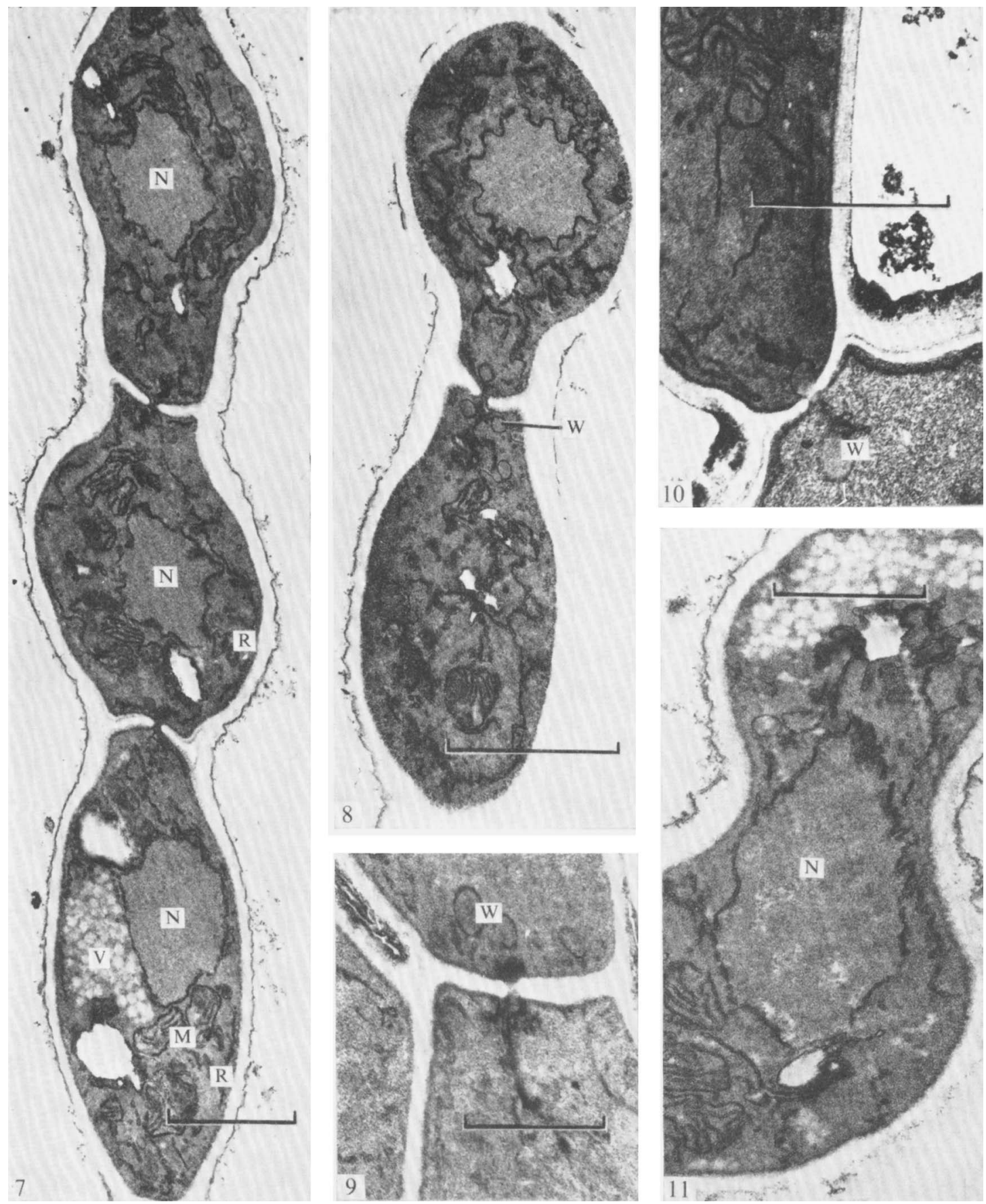

Fig. 7. Mutant 22B. Longitudinal section of a pseudo-conidial chain. Each unit contains a nucleus, $\mathrm{N}$, mitochondria, $\mathrm{M}$, endoplasmic reticulum, $\mathrm{R}$, and a region of transparent vacuoles $\mathrm{V}$; note the septal pores connecting the successive units.

Fig. 8. The two last elements of a pseudo-conidial chain. The wall is single-layered, without differential thickening. The septal pore is bordered by endoplasmic reticulum and Woronin bodies, W.

Fig. 9. Detail of the connexion between metula and phialide.

Fig. Io. Detail of the connexion between vesicle and metula.

Fig. II. The constriction zone between two apical pseudo-conidia before nuclear division and septum formation.

(Bar markers represent $\mathrm{I} \mu \mathrm{m}$.) 

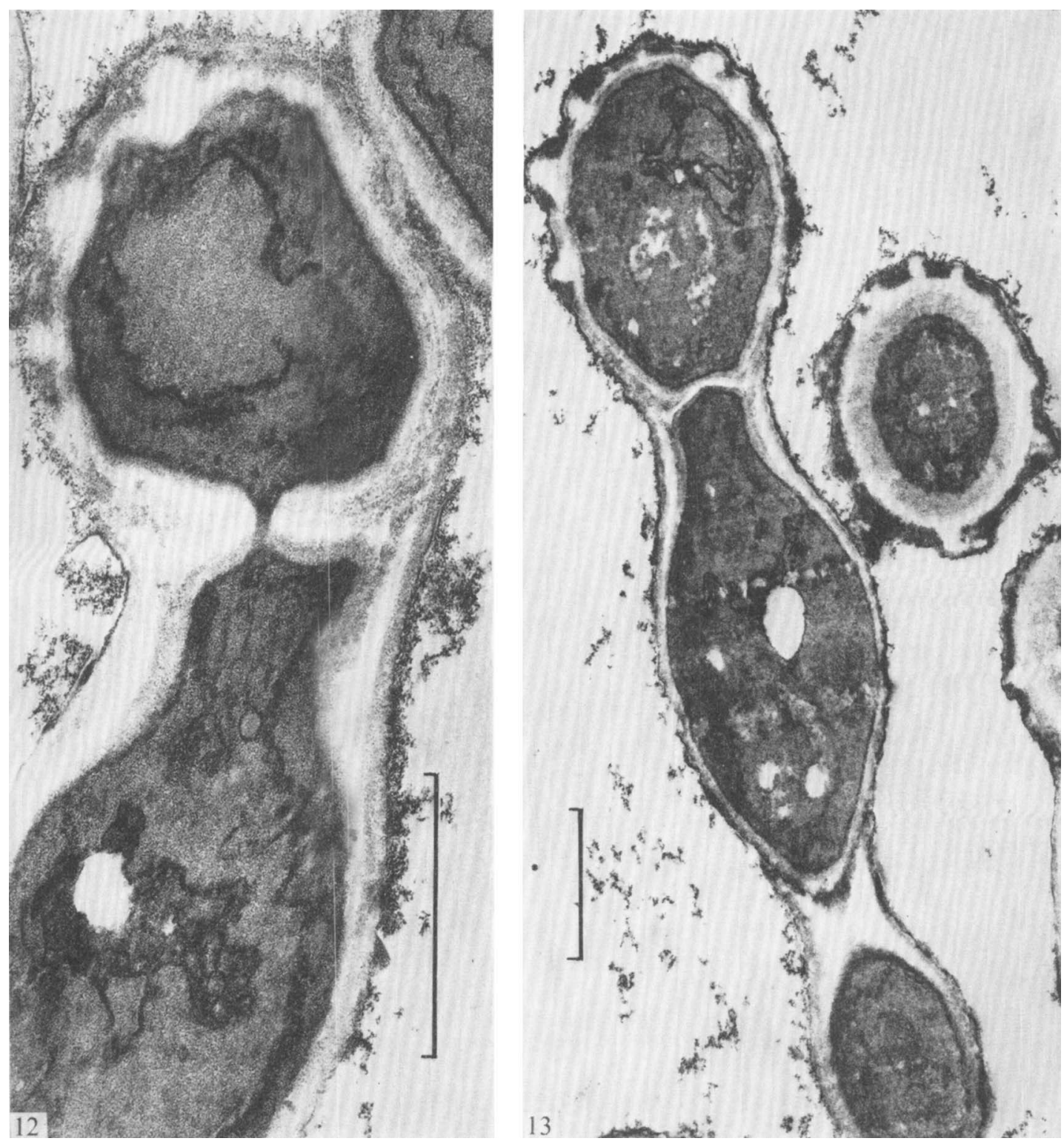

Fig. I 2 and 13 . Mutant 22B. Terminal units of a pseudo-conidial chain turning into true conidia; note the differential wall thickening and occlusion of the septal pore.

(Bar markers represent I $\mu \mathrm{m}$.)

\section{DISCUSSION}

Sporulation in wild-type Aspergillus aureolatus was similar to the process described by other authors in Aspergillus (Trinci et al. I968).

The behaviour of the temperature-sensitive and osmotic-remedial mutant 22B was the same as that of the 'abacus' mutants studied by Clutterbuck (1969) in Aspergillus nidulans. 
The development of true conidia was blocked, and there was a hypha-like process of apical extension at the phialide tip. Before the bud growing at the phialide tip had reached full size, it resumed the function of a sterigma and initiated budding from its apex once more. Our ultrastructural study showed that the structures formed by mutant 22B under conditions non-permissive for normal conidiation should be regarded as pseudo-phialides rather than pseudo-conidia, because their walls were thin, their septal pores remained open, and they grew apically. However, it should be pointed out that each apical element was at the same time a potential conidium, because at lower temperatures it could develop into a true conidium: its wall completed the process of thickening.

The results of the present study do not substantiate a theory proposed by Raper \& Fennell (1965) to explain the behaviour of a similar mutant of Aspergillus niger which Yuill \& Yuill (1938) described as Cladosarum olivaceum. They suggested that in this mutant the 'dormant' nucleus remained in the phialide whilst the 'active' nucleus migrated into a newly formed 'conidium'. This nuclear differentiation could be responsible for the repeated sequence of cells functioning as phialides. However, this hypothesis implies a determinism which cannot account for the reversion of the young phialide-like elements, already formed, to become normal conidia once the temperature was lowered. We presume that there is no definite difference between the two daughter-nuclei, but that the defect has to be sought in the wall of the mutant, which could not be completely synthesized at non-permissive conditions. Our data support the contention that the phenotypical reversal of the temperaturesensitive defect is linked with wall-disfunctions.

\section{REFERENCES}

Border, D. J. \& Trinci, A. P. J. (1970). Fine structure of the germination of Aspergillus nidulans conidia. Transactions of the British Mycological Society 54, 143-152.

CAmpbell, C. K. (I97I). Fine structure and physiology of conidial germination in Aspergillus fumigatus. Transactions of the British Mycological Society 57, 393-402.

Clutterbuck, A. J. (1969). A mutational analysis of conidial development in Aspergillus nidulans. Genetics 63, $317-327$.

Florance, E. R., Denison, W. C. \& Allen, T. C. (1972). Ultrastructure of dormant and germinating conidia of Aspergillus nidulans. Mycologia 64, I I 5-123.

Muntanjola-Cvetković, M. \& Nešković, M. (1972a). Physiological properties of the Cladosarum-like mutant 22B of Aspergillus aureolatus. Archiv für Mikrobiologie 84, 14I-I52.

Muntanjola-Cvetković, M. \& Nešković, M. (1972b). A Cladosarum-like mutant of Aspergillus aureolatus that is temperature-sensitive and osmotic-remedial. Genetika, acta biologica iugoslavica 4, 305-3I 2.

RAPER, K. B. \& FenNell, D. I. (1965). The Genus Aspergillus. Baltimore: Williams \& Wilkins.

TANAKA, K. (1966). Change in ultrastructure of Aspergillus oryzae conidia during germination. Journal of General and Applied Microbiology r2, 239-246.

TANAKA, K. \& YANAGITA, T. (I963). Electron microscopy of ultrathin sections of Aspergillus niger. II. Fine structure of conidia-bearing apparatus. Journal of General and Applied Microbiology 9, I89-203.

Trinci, A. P. J., Peat, A. \& Banbury, G. H. (i968). Fine structure of phialide and conidiospore development in Aspergillus giganteus Wehmer. Annals of Botany 32, 24I-250.

Tsukahara, T. (1968). Electron microscopy of germinating conidiospores of Aspergillus niger. Sabouraudia 6, I $85-\mathrm{I} 9 \mathrm{I}$.

Weisberg, S. H. \& Turian, G. (197I). Ultrastructure of Aspergillus nidulans conidia and conidial lomasomes. Protoplasma 72, 55-67.

Yuill, E. \& YuILl, J. L. (1938). Cladosarum olivaceum, a new Hyphomycete. Transactions of the British Mycological Society 22, 194-200. 